PROCEEDINGS OF THE

AMERICAN MATHEMATICAL SOCIETY

Volume 134, Number 5, Pages 1483-1493

S 0002-9939(05)08112-8

Article electronically published on October 5, 2005

\title{
AVOIDING THE PROJECTIVE HIERARCHY IN EXPANSIONS OF THE REAL FIELD BY SEQUENCES
}

\author{
CHRIS MILLER
}

(Communicated by Carl G. Jockusch, Jr.)

\begin{abstract}
Some necessary conditions are given on infinitely oscillating real functions and infinite discrete sets of real numbers so that first-order expansions of the field of real numbers by such functions or sets do not define $\mathbb{N}$. In particular, let $f: \mathbb{R} \rightarrow \mathbb{R}$ be such that $\lim _{x \rightarrow+\infty} f(x)=+\infty, f(x)=O\left(e^{x^{N}}\right)$ as $x \rightarrow+\infty$ for some $N \in \mathbb{N},(\mathbb{R},+, \cdot, f)$ is o-minimal, and the expansion of $(\mathbb{R},+, \cdot)$ by the set $\{f(k): k \in \mathbb{N}\}$ does not define $\mathbb{N}$. Then there exist $r>0$ and $P \in \mathbb{R}[x]$ such that $f(x)=e^{P(x)}\left(1+O\left(e^{-r x}\right)\right)$ as $x \rightarrow+\infty$.
\end{abstract}

We are interested in expansions, in the sense of first-order definability, of the real field $\overline{\mathbb{R}}:=(\mathbb{R},+, \cdot)$ that define infinitely oscillating functions or infinite discrete sets but do not define the set of non-negative integers $\mathbb{N}$; see 12 for context and extended discussion. In this paper, some necessary conditions are developed for some special, but natural, cases. A bit of work is required before the main results can be stated in full, but preliminary versions can be given right away.

An expansion of $\overline{\mathbb{R}}$ is o-minimal if every definable subset of $\mathbb{R}$ is a finite union of points and open intervals. (Throughout, "definable" means "definable with parameters".) The literature on o-minimal expansions of $\overline{\mathbb{R}}$ has become extensive; see $[3,4,5$, for general reference and surveys.

For each fixed $c \in \mathbb{R}$, the expansion of $\overline{\mathbb{R}}$ by the function $\sin (c \log x)$ does not define $\mathbb{N}$ (indeed, much more is true; see 1.10 below for details). A preliminary statement of the first main result of this paper shows that we cannot do much better.

Theorem A. Let $f: \mathbb{R} \rightarrow \mathbb{R}$ be such that $f$ is bounded below as $x \rightarrow+\infty$ by a compositional iterate of $\log x$. If $(\overline{\mathbb{R}}, f)$ is o-minimal and $(\overline{\mathbb{R}}, \sin f)$ does not define $\mathbb{N}$, then there exist $a, b, c, r \in \mathbb{R}$ and $g: \mathbb{R} \rightarrow \mathbb{R}$ such that $c, r>0, \lim _{x \rightarrow+\infty} x^{r} g(x)=$ 1 and $f(x)=c \log x+a+b g(x)$ for all sufficiently large $x$.

(See Theorem 2.2 for the full statement.) The converse fails, but details are postponed. It is not yet known if there exist $f$ such that $(\overline{\mathbb{R}}, f)$ is o-minimal, $\lim _{x \rightarrow+\infty} f(x)=+\infty$ and $f$ is not bounded below as $x \rightarrow+\infty$ by a compositional iterate of $\log x$.

Definable oscillatory functions tend to give rise to definable infinite sequences, and in any case, there are infinite sequences that are of independent interest. For

Received by the editors February 4, 2004 and, in revised form, November 17, 2004.

2000 Mathematics Subject Classification. Primary 03C64; Secondary 26 A12.

This research was partially supported by NSF Grant No. DMS-9988855.

(C)2005 American Mathematical Society Reverts to public domain 28 years from publication 
example, given $P \in \mathbb{Z}[x]$, the expansion of $\overline{\mathbb{R}}$ by the set $\left\{2^{P(n)}: n \in \mathbb{N}\right\}$ does not define $\mathbb{N}$ (again, more is true; see 1.10). A preliminary statement of our second main result shows that we cannot do much better.

Theorem B. Let $f: \mathbb{R} \rightarrow \mathbb{R}$ be such that $\lim _{x \rightarrow+\infty} f(x)=+\infty$ and $f(x) \leq e^{x^{N}}$ for some $N \in \mathbb{N}$ and all sufficiently large $x$. If $(\overline{\mathbb{R}}, f)$ is o-minimal and the expansion $(\overline{\mathbb{R}}, f(\mathbb{N}))$ of $\overline{\mathbb{R}}$ by the set $\{f(k): k \in \mathbb{N}\}$ does not define $\mathbb{N}$, then there exist $c, r>0$ and $P \in \mathbb{R}[x]$ such that $\left|f(x) e^{-P(x)}-1\right| \leq c e^{-r x}$ for all sufficiently large $x$.

(See Theorem 3.2 for the full statement.) The conclusion is that $f$ is asymptotic to $e^{P}$ at $+\infty$ in a strong sense (as opposed to just $\lim _{x \rightarrow+\infty} f(x) e^{-P(x)}=1$ ). Again, the converse fails; details are postponed.

Corollary (of Theorem B). Let $f: \mathbb{R} \rightarrow \mathbb{R}$ be such that $\lim _{x \rightarrow+\infty} f(x)=+\infty$ and $(\overline{\mathbb{R}}, f)$ is o-minimal. If $(\overline{\mathbb{R}}, f(\mathbb{N}))$ does not define $\mathbb{N}$, then $(\overline{\mathbb{R}}, f)$ defines the function $e^{x}$. If $\left(\overline{\mathbb{R}}, e^{x}, f(\mathbb{N})\right)$ does not define $\mathbb{N}$, then $f$ is not bounded above as $x \rightarrow+\infty$ by any fixed compositional iterate of $e^{x}$.

Proof. The first assertion follows from the growth dichotomy for o-minimal expansions of $\overline{\mathbb{R}}([11]$, restated below in $\S 1)$. For the second, note that for each fixed compositional iterate $L$ of $\log x$ (with $\log x:=0$ for $x \leq 0$ ), the set $\{L(f(k)): k \in \mathbb{N}\}$ is definable in $\left(\overline{\mathbb{R}}, e^{x}, f(\mathbb{N})\right)$.

Again, it is not yet known if there exist $f: \mathbb{R} \rightarrow \mathbb{R}$ such that $(\overline{\mathbb{R}}, f)$ is o-minimal and $f$ is not bounded above as $x \rightarrow+\infty$ by some iterate of $e^{x}$.

Both theorems are connected with the study of expansions of $\overline{\mathbb{R}}$ by cyclic subgroups of $\left(\mathbb{R}^{>0}, \cdot\right)$; see Theorem 2.2 and Corollary 3.3

The theorems and connections to cyclic multiplicative groups rest on a simple principle that applies in much greater generality; it is worth pointing out and proving now.

Asymptotic Extraction of Groups (AEG, for short). An expansion of $\overline{\mathbb{R}} d e-$ fines $\mathbb{N}$ iff it defines the range of a sequence $\left(a_{k}\right)_{k \in \mathbb{N}}$ of real numbers such that $\lim _{k \rightarrow+\infty}\left(a_{k+1}-a_{k}\right) \in \mathbb{R} \backslash\{0\}$. For $\alpha>0$, an expansion of $\overline{\mathbb{R}}$ defines the set $\left\{\alpha^{k}: k \in \mathbb{Z}\right\}$ iff it defines the range of a sequence $\left(a_{k}\right)_{k \in \mathbb{N}}$ of nonzero real numbers such that $\lim _{k \rightarrow+\infty} a_{k+1} / a_{k}=\alpha$.

Proof. Let $\left(a_{k}\right)_{k \in \mathbb{N}}$ be a sequence of real numbers and put $A=\left\{a_{k}: k \in \mathbb{N}\right\}$.

Suppose that $\lim _{k \rightarrow+\infty}\left(a_{k+1}-a_{k}\right)=\alpha \neq 0$. By replacing $A$ with $\{-a: a \in A\}$, we take $\alpha>0$. Then $\lim _{k \rightarrow+\infty} a_{k}=+\infty$, and $\lim _{k \rightarrow+\infty}\left(a_{k+j}-a_{k}\right)=\alpha j$ for each fixed $j \in \mathbb{Z}$, so

$$
\alpha \mathbb{Z}=\{r \in \mathbb{R}: \forall \epsilon, M>0 \exists x, y \in A \cap(M, \infty),|x-y-r|<\epsilon\} .
$$

Hence, $(\overline{\mathbb{R}}, A)$ defines $\mathbb{N}$. (Aside: We have actually shown that $(\mathbb{R},<,+, A)$ defines $\alpha \mathbb{Z}$.)

The proof of the multiplicative version is similar; details are left to the reader.

Here is an outline of the rest of the paper. In $\$ 1$ some notation, conventions and key basic technical results are established. Theorem A is treated in $₫ 2$, Theorem B is dealt with in a more general setting, namely, that of Hardy fields; we do this in 33 . 


\section{Preliminaries}

"Reduct" and "expansion" are always used in the sense of definability (as opposed to syntax). Given structures $\mathfrak{R}$ and $\mathfrak{R}^{\prime}$, we write $\mathfrak{R}=\mathfrak{R}^{\prime}$ if they are interdefinable. Denote $(\overline{\mathbb{R}}, \mathbb{N})$ by PH, short for "(real) projective hierarchy"; see e.g. 9 , $\S 37 . \mathrm{A}]$, for basic facts about PH. Every countable subset of $\mathbb{R}$ is definable in $\mathrm{PH}$, so $(\overline{\mathbb{R}},\langle f\rangle)$ is a reduct of $\mathrm{PH}$. Hence, $(\overline{\mathbb{R}},\langle f\rangle)=\mathrm{PH}$ if and only if $(\overline{\mathbb{R}},\langle f\rangle)$ defines $\mathbb{N}$.

The variables $k, m, n$ range over $\mathbb{N}$ (the non-negative integers). Sequences and series are indexed by $\mathbb{N}$ unless indicated otherwise. Functions are $\mathbb{R} \rightarrow \mathbb{R}$ unless indicated otherwise; $f$ and $g$ range over functions. The restriction of $f$ to $X \subseteq \mathbb{R}$ is denoted by $f\lceil X$. A function is infinitely increasing if it is ultimately strictly increasing and unbounded above; ultimately abbreviates "for all sufficiently large positive arguments". We write $f \sim g$ if, for each fixed $\epsilon>0$, ultimately $|f-g| \leq$ $\epsilon|g|$. (Of course, if $g$ ultimately has no zeros, then $f \sim g$ iff $\lim _{t \rightarrow+\infty} f(t) / g(t)=1$.) The difference operator $f \mapsto f(x+1)-f$ is denoted by $\Delta$, its $n$-th iterate by $\Delta^{n}$. (Throughout, $f(x+1)$ means $f \circ(x+1)$, not $f \cdot(x+1)$.) The definitions are relativized to functions $\mathbb{N} \rightarrow \mathbb{R}$ and sequences of real numbers in the obvious way.

Let $\langle f\rangle$ denote the image of $\mathbb{N}$ under $f$. Typically, we regard $\langle f\rangle$ as a germ at $+\infty$, i.e., we identify $\langle f\rangle$ and $\langle g\rangle$ if $f(k)=g(k)$ for all but finitely many $k$. We say that $\langle f\rangle$ is constant (or monotone, or infinitely increasing) if the same is true ultimately of $f \nmid \mathbb{N}$; we write $\langle f\rangle \sim\langle g\rangle$ if $f(k) \sim g(k)$, and so on. Obviously, $(\overline{\mathbb{R}}, g,\langle f\rangle)$ defines $\langle g \circ f\rangle$.

Note the following; it will be used frequently.

1.1. If $\langle f\rangle$ is strictly monotone and $N \in \mathbb{N}$ is fixed, then $(\mathbb{R},\langle f\rangle)$ defines the sequences $\langle f(x+N)\rangle,\left\langle\Delta^{N} f\right\rangle$ and $\left\langle\exp \left(\Delta^{N} \log |f|\right)\right\rangle$.

Proof. We may assume that $\langle f\rangle$ is positive. Then, ultimately, $f(n+N)$ is the $N$-th successor of $f(n)$ in $(\langle f\rangle,<)$. Since $N$ is fixed, $(\mathbb{R},<,\langle f\rangle)$ defines $\langle f(x+N)\rangle$. An easy induction shows that $\Delta^{N} f$ is a $\mathbb{Z}$-linear combination of the $f(x+k), k=$ $0, \ldots, N$, so $(\mathbb{R},<,+,\langle f\rangle)$ defines $\left\langle\Delta^{N} f\right\rangle$, and $(\mathbb{R},<, \cdot,\langle f\rangle)$ defines $\exp \left(\Delta^{N} \log f\right)$.

Next is a key intermediate result.

1.2. Let $f, g:(a, \infty) \rightarrow \mathbb{R}$ be infinitely increasing and $C^{1}$ with positive derivatives such that $g^{\prime} \sim f^{\prime}$. Then $\left(\overline{\mathbb{R}}, g,\left\langle f^{-1}\right\rangle\right)=\mathrm{PH}$, where $f^{-1}$ denotes the compositional inverse of $f$.

Proof. Then $\left\langle\Delta\left(g \circ f^{-1}\right)\right\rangle \sim 1$ by the Mean Value Theorem. Apply AEG.

For $\alpha>0$, put $\alpha^{\mathbb{Z}}=\left\{\alpha^{j}: j \in \mathbb{Z}\right\}$. Note that $\alpha^{\mathbb{Z}},\left\langle\alpha^{x}\right\rangle$ and $\left\langle\alpha^{-x}\right\rangle$ are pairwise interdefinable over $\overline{\mathbb{R}}$.

In general, there is no reason to think that $\langle f+g\rangle$ or $\langle f g\rangle$ are definable in $(\overline{\mathbb{R}},\langle f\rangle,\langle g\rangle)$. But this, and more, is true in an important special case:

1.3. Let $\alpha>0,\langle f\rangle \sim\left\langle\alpha^{x}\right\rangle$ and $h: \mathbb{R}^{2} \rightarrow \mathbb{R}$. Then $(\overline{\mathbb{R}}, h,\langle f\rangle)$ defines $\left\langle h \circ\left(f, \alpha^{x}\right)\right\rangle$; in particular, $(\overline{\mathbb{R}},\langle f\rangle)$ defines $\alpha^{\mathbb{Z}}$.

Proof. It suffices to show that $(\overline{\mathbb{R}},\langle f\rangle)$ defines $\left\{\left(f(k), \alpha^{k}\right): k \in \mathbb{N}\right\}$. We may assume that $\alpha>1$; then $\langle f\rangle$ is infinitely increasing. Now, $\langle f(x+1)\rangle \sim\left\langle\alpha^{x+1}\right\rangle=$ $\left\langle\alpha \alpha^{x}\right\rangle \sim\langle\alpha f\rangle$, so $(\overline{\mathbb{R}},\langle f\rangle)$ defines $\alpha^{\mathbb{Z}}$ by AEG. Choose $\epsilon \in(0,1)$ such that $\alpha>$ 
$(1+\epsilon) /(1-\epsilon)$, then choose $M$ such that $(1-\epsilon) \alpha^{k}<f(k)<(1+\epsilon) \alpha^{k}$ for all $k>M$. Then for all $m, n>M$ we have $m=n$ iff $(1-\epsilon) \alpha^{n}<f(m)<(1+\epsilon) \alpha^{n}$.

1.4. If $f$ is infinitely increasing and $\lim _{k \rightarrow+\infty}[\log f(k)-P(k)]=0$ for some $P \in$ $\mathbb{R}[x]$, then there exists $\alpha>1$ such that $(\overline{\mathbb{R}},\langle f\rangle)$ defines $\alpha^{\mathbb{Z}}$.

Proof. Put $d=\operatorname{deg} P$. Then $\Delta^{d-1} P=a x+b$ for some $a, b \in \mathbb{R}$ with $a>0$, and $\left\langle\exp \left(\Delta^{d-1} \log f\right)\right\rangle \sim\left\langle e^{b} e^{a x}\right\rangle$. Recall that $(\overline{\mathbb{R}},\langle f\rangle)$ defines $\left\langle\exp \left(\Delta^{d-1} \log f\right)\right\rangle$ and apply 1.3 .

For $r \in \mathbb{R}, x^{r}$ denotes the power function $t \mapsto t^{r}:(0, \infty) \rightarrow \mathbb{R}$; for convenience, we put $t^{r}=0$ for $t \leq 0$. Given an expansion $\mathfrak{R}$ of $\mathbb{R}$, the set of all $r \in \mathbb{R}$ such that $\mathfrak{R}$ defines $x^{r}$ is a field, called the field of exponents of $\mathfrak{R}$. It is an easy consequence of the Tarski-Seidenberg theorem that $\overline{\mathbb{R}}$ is o-minimal and has field of exponents $\mathbb{Q}$. Given $S \subseteq \mathbb{R},\left(\overline{\mathbb{R}},\left(x^{r}\right)_{r \in S}\right)$ has field of exponents $\mathbb{Q}(S)$ [10]. Since $\left(\overline{\mathbb{R}}, e^{x}\right)$ defines $\log$, it has field of exponents $\mathbb{R}$.

1.5. Let $\mathfrak{R}$ be an expansion of $\overline{\mathbb{R}}$ such that every definable subset of $\mathbb{R}$ either has interior or is nowhere dense. Then either $\mathfrak{R}$ defines no proper nontrivial subgroups of $\left(\mathbb{R}^{>0}, \cdot\right)$ or $\mathfrak{R}$ has field of exponents $\mathbb{Q}$ and there exists $\alpha>1$ such that every proper subgroup of $\left(\mathbb{R}^{>0}, \cdot\right)$ definable in $\mathfrak{R}$ is of the form $\alpha^{q \mathbb{Z}}$ for some $q \in \mathbb{Q}$.

Proof. If $\alpha, \beta>1$ and $\beta$ is not a rational power of $\alpha$, then the direct product $\alpha^{\mathbb{Z}} \cdot \beta^{\mathbb{Z}}$ is both dense and codense in the positive real line.

1.6. At present, very little is known about expansions of $\overline{\mathbb{R}}$ by collections of groups $\alpha^{\mathbb{Z}}$ (unless all of the groups are rational power images of one of them), even in concrete cases like $\left(\overline{\mathbb{R}}, 2^{\mathbb{Z}}, 3^{\mathbb{Z}}\right)$, except that dense-codense subsets of $\mathbb{R}$ are definable (1.5); in particular, it is not yet known if such structures can define $\mathbb{N}$. Similarly, very little is known about the structures $\left(\overline{\mathbb{R}}, x^{r}, \alpha^{\mathbb{Z}}\right)$ where $r$ is irrational and $\alpha>1$ (since $\alpha^{r \mathbb{Z}}$ is definable). The scope of some of the results of this paper is limited by this lack of knowledge.

Given a sequence $\left(f_{n}\right)$ of functions, we write $f \sim \sum f_{n}$ if for each $m$ and $\epsilon>0$, ultimately $\left|f-\sum_{n=0}^{m} f_{n}\right| \leq \epsilon\left|f_{m}\right|$. Warning: Suppose that a series $F:=\sum f_{n}$ of functions converges to a function on some ray $(a, \infty)$, in particular, if $f_{n} \uparrow(a, \infty)=$ 0 for all but finitely many $n$. Then the meaning of " $f \sim F$ " depends on whether $F$ is regarded as a function or a formal series. This ambiguity occurs often in the literature on asymptotic analysis but causes no trouble in practice provided that sufficient context is given.

Let $\mathbb{R}\left(\left(x^{\mathbb{R}}\right)\right)_{\omega}$ denote the set of all series $\sum c_{n} x^{r_{n}}$, where $\left(r_{n}\right)$ is a strictly decreasing sequence of real numbers; we do not require that $r_{n} \rightarrow-\infty$. The support of $\sum c_{n} x^{r_{n}}$, denoted by $\operatorname{supp}\left(\sum c_{n} x^{r_{n}}\right)$, is the set $\left\{r_{n}: c_{n} \neq 0\right\}$. For $S \subseteq \mathbb{R}$, let $\mathbb{R}\left(\left(x^{S}\right)\right)_{\omega}$ denote the set of all $F \in \mathbb{R}\left(\left(x^{\mathbb{R}}\right)\right)_{\omega}$ such that $\operatorname{supp}(F) \subseteq S$. Given $F=\sum c_{n} x^{r_{n}} \in \mathbb{R}\left(\left(x^{\mathbb{R}}\right)\right)_{\omega}$, the series $\sum c_{n} g^{r_{n}}$ is denoted by $F \circ g$ or $F(g)$ as convenient.

1.7. If $f \sim F \in \mathbb{R}\left(\left(x^{\mathbb{R}}\right)\right)_{\omega}$, then $\operatorname{supp}(F)$ is contained in the field of exponents of $(\overline{\mathbb{R}}, f)$.

Proof. If $f \sim c x^{r}, c \neq 0$ and $s>0$, then $s^{r}=\lim _{t \rightarrow+\infty} f(s t) / f(t)$. Since $\operatorname{supp}(F)$ is well ordered, the rest is an easy induction.

A routine induction based on 1.3 yields the following discrete version of 1.7 
1.8. Let $\alpha>0, \sum c_{n} x^{r_{n}} \in \mathbb{R}\left(\left(x^{\mathbb{R}}\right)\right)_{\omega}, r \in \operatorname{supp}\left(\sum c_{n} x^{r_{n}}\right)$ and $d \in \mathbb{N}$. If $f(k) \sim$ $\sum c_{n} \alpha^{r_{n} k}$, then $(\overline{\mathbb{R}},\langle f\rangle)$ defines both $\alpha^{r \mathbb{Z}}$ and $\left\langle f-\sum_{n \leq d} c_{n} \alpha^{r_{n} x}\right\rangle$.

Next, we recall two key results from o-minimality.

Monotonicity Theorem. If $(\overline{\mathbb{R}}, f)$ is o-minimal, then there is a partition of $\mathbb{R}$ into finitely many points and open intervals such that, for each interval $I$ of the partition, $f\left\lceil I\right.$ is $C^{1}$ and either constant or strictly monotone.

(See e.g. 3] for the proof.)

An expansion of $\overline{\mathbb{R}}$ is polynomially bounded if for each definable $f$ there exists $n$ (depending on $f$ ) such that ultimately $f \leq x^{n}$, and is exponential if it defines the function $e^{x}$.

Growth Dichotomy ([11]). Every o-minimal expansion of $\overline{\mathbb{R}}$ is either exponential or polynomially bounded. If $f$ is definable in a polynomially bounded o-minimal expansion of $\overline{\mathbb{R}}$ with field of exponents $K$, then there exists $F \in \mathbb{R}\left(\left(x^{K}\right)\right)_{\omega}$ such that either $\operatorname{supp}(F)$ is infinite and $f \sim F$, or $\operatorname{supp}(F)$ is finite and ultimately $f=F$.

For some interesting examples of polynomially bounded o-minimal expansions of $\overline{\mathbb{R}}$, see $[6,7,10,15$.

An expansion $\mathfrak{R}$ of $(\mathbb{R},<)$ is d-minimal (short for "discrete-minimal") if for every $\mathfrak{R}^{\prime}$ elementarily equivalent to $\mathfrak{R}$, every unary set definable in $\mathfrak{R}^{\prime}$ either has interior or is a finite union of discrete sets. (The idea is that d-minimality should be the next best thing to o-minimality, so to speak, for expansions of $\overline{\mathbb{R}}$ that define infinite discrete sets.) If $\mathfrak{R}$ is d-minimal, then every subset of $\mathbb{R}$ definable in $\mathfrak{R}$ either has interior or is nowhere dense - much more can be said; see 12 - so $\mathfrak{R} \neq \mathrm{PH}$. Next, we consider some examples.

1.9. Let $\mathfrak{R}$ be an o-minimal expansion of $\overline{\mathbb{R}}, \alpha>1$, and let $f$ be such that any of the following hold:

(a) $\langle f\rangle \subseteq \mathbb{Z}$ and $\mathfrak{R}$ has field of exponents $\mathbb{Q}$.

(b) There exists $\beta>1$ such that $\langle f\rangle \subseteq \beta^{\mathbb{N}}$ and $\left(\Re, x^{\beta}\right)$ is polynomially bounded.

(c) $\lim _{k \rightarrow+\infty} f(k)=+\infty=\lim _{k \rightarrow+\infty} f(k+1) / f(k)$ and $\mathfrak{R}$ is polynomially bounded.

Then $\left(\Re,\left\langle\alpha^{f}\right\rangle\right)$ is d-minimal. See [12, §3.4] for (a), [14] for (b), and [8] for (c).

1.10. Some instances of 1.9(a) are particularly relevant to this paper. First, $\left(\overline{\mathbb{R}},\left\langle\alpha^{P}\right\rangle\right)$ is d-minimal for any $\alpha>0$ and $P \in \mathbb{Z}[x]$, so Theorem $\mathrm{B}$ is not vacuous. (I do not yet know what happens in general for $P \in \mathbb{R}[x]$.) Next we show that Theorem $\mathrm{A}$ is not vacuous. Let $c>0$; we show that the expansion of $\overline{\mathbb{R}}$ by the function $\sin (c \log x)$ is d-minimal. Put $\alpha=e^{2 \pi / c}$ and $h=\sin (c \log x) \uparrow[1, \alpha]$. By [2], $(\overline{\mathbb{R}}, h)$ is o-minimal and has field of exponents $\mathbb{Q}$. By $1.9(\mathrm{a}),\left(\overline{\mathbb{R}}, h, \alpha^{\mathbb{Z}}\right)$ is d-minimal. Now observe that $\left(\overline{\mathbb{R}}, h, \alpha^{\mathbb{Z}}\right)$ defines $\sin (c \log x)$ : For $x>0$, we have $y=\sin (c \log x)$ iff there exist $a \in \alpha^{\mathbb{Z}}$ and $b \in[1, \alpha)$ such that $x=a b$ and $y=h(b)$.

We close this section with a technical

1.11. Proposition. Let $f:(a, \infty) \rightarrow \mathbb{R}$ be differentiable and bounded below by $a$ compositional iterate of $\log x$, let $(\overline{\mathbb{R}}, f)$ be o-minimal and let $\left(\overline{\mathbb{R}}, f^{\prime},\left\langle f^{-1}\right\rangle\right) \neq \mathrm{PH}$. 
Then:

(a) There exist $c>0$ and $F \in \mathbb{R}\left(\left(x^{\mathbb{R}}\right)\right)_{\omega}$ such that $\operatorname{supp}(F) \subseteq(-\infty, 0]$, and either $\operatorname{supp}(F)$ is infinite and $f \sim c \log x+F$, or $\operatorname{supp}(F)$ is finite and ultimately $f=c \log x+F$.

(b) $\left(\overline{\mathbb{R}},\left\langle f^{-1}\right\rangle\right)$ defines $\left(e^{1 / c}\right)^{\mathbb{Z}}$.

(c) If every subset of $\mathbb{R}$ definable in $\left(\overline{\mathbb{R}}, f^{\prime},\left\langle f^{-1}\right\rangle\right)$ either has interior or is nowhere dense, then $\left(\overline{\mathbb{R}}, f^{\prime},\left\langle f^{-1}\right\rangle\right)$ has field of exponents $\mathbb{Q}$ and $F \in \mathbb{R}\left(\left(x^{\mathbb{Q}}\right)\right)_{\omega}$.

Proof. (a). By the Monotonicity Theorem, we may assume that $f^{\prime}$ has no zeros. By assumption, $f$ is bounded below by some compositional iterate $L$ of $\log x$; by iterating $L$ further, we may assume that $f / L$ is infinitely increasing, so the same is true of $f^{\prime} / L^{\prime}$. By asymptotic integration (e.g. [13, Prop. 4]), there exists $g$ definable in $\left(\overline{\mathbb{R}}, f^{\prime}, e^{x}\right)$ such that $g^{\prime} \sim f^{\prime}$. Hence, by 1.2 and growth dichotomy, there exist $c>0, r \in \mathbb{R}$ and $G \in \mathbb{R}\left(\left(x^{\mathbb{R}}\right)\right)_{\omega}$ such that $\operatorname{supp}(G) \subseteq(-\infty, r)$, and either $\operatorname{supp}(G)$ is infinite and $f^{\prime} \sim c x^{r}+G$, or $\operatorname{supp}(G)$ is finite and ultimately $f^{\prime}=c x^{r}+G$. Then $r=-1$ (for if not, then $g:=c x^{r+1} /(r+1)$ is definable in $\left(\overline{\mathbb{R}}, f^{\prime}\right)$ by 1.7] and $g^{\prime} \sim f^{\prime}$, contradicting 1.2$)$. To obtain $F$, add an appropriate real constant to the formal antiderivative of $G$.

(b). Since $f^{\prime} \sim c / x$, we have $\left(\log f^{-1}\right)^{\prime} \sim 1 / c$, so $f^{-1}(x+1) \sim e^{1 / c} f^{-1}$. Apply AEG.

(c). Immediate from (b), 1.5 and 1.7 .

1.12. Proposition 1.11 is not vacuous: By $1.9(\mathrm{a}),\left(\mathfrak{R}, \alpha^{\mathbb{N}}\right)$ is d-minimal for any $\alpha>$ 1 and o-minimal expansion $\mathfrak{R}$ of $\overline{\mathbb{R}}$ having field of exponents $\mathbb{Q}$, so the hypothesis of the proposition holds with $f=\log _{\alpha} x$.

1.13. The converse of Proposition 1.11 fails. Put $f=\log x+x /(x-1)+e^{-x}$; then $f \sim \log x+\sum x^{-k}$ and $(\overline{\mathbb{R}}, f)$ is o-minimal, indeed, $(\overline{\mathbb{R}}, f)=\left(\overline{\mathbb{R}}, e^{x}\right)=\left(\overline{\mathbb{R}}, f^{\prime}\right)$. By Proposition $1.11(\mathrm{~b}),\left(\overline{\mathbb{R}},\left\langle f^{-1}\right\rangle\right)$ defines $e^{\mathbb{Z}}$. Hence, $\left(\overline{\mathbb{R}}, f^{\prime},\left\langle f^{-1}\right\rangle\right)=$ PH. If we require moreover that $\left(\overline{\mathbb{R}}, f^{\prime}\right)$ be polynomially bounded, then the situation is unclear. For one thing, we run into trouble immediately if $\operatorname{supp}(F) \nsubseteq \mathbb{Q}$ : By 1.7 and Proposition 1.11(b), ( $\left.\overline{\mathbb{R}}, f^{\prime},\left\langle f^{-1}\right\rangle\right)$ defines both an irrational power $x^{r}$ and a nontrivial $\alpha^{\mathbb{Z}}$; now recall 1.6. Even if we assume that $\left(\overline{\mathbb{R}}, f^{\prime}\right)$ has field of exponents $\mathbb{Q}$, it is not yet clear what can be said, for although $\left(\overline{\mathbb{R}}, f^{\prime}, \alpha^{\mathbb{Z}}\right)$ is then d-minimal, it is not yet known how to control the interaction of $\alpha^{\mathbb{Z}}$ with $\left\langle f^{-1}\right\rangle$.

\section{EXPANDING BY OSCILLATIONS}

We now obtain a stronger version of Theorem A. First, we make an easy observation, whose proof we leave to the reader.

2.1. Let $I \subseteq \mathbb{R}$ be an open interval and let $f: I \rightarrow \mathbb{R}$ be $C^{1}$ such that $f^{\prime}$ has no zeros. Then $(\overline{\mathbb{R}}, \sin f)=\left(\overline{\mathbb{R}}, \cos f, f^{\prime}\right)=\left(\overline{\mathbb{R}}, \sin f, f^{\prime}\right)=(\overline{\mathbb{R}}, \cos f)$.

2.2. Theorem. Suppose that $(\overline{\mathbb{R}}, \sin f) \neq \mathrm{PH},(\overline{\mathbb{R}}, f)$ is o-minimal and $f$ is ultimately bounded below by some compositional iterate of $\log x$. Then:

(a) There exist $c>0$ and $F \in \mathbb{R}\left(\left(x^{\mathbb{R}}\right)\right)_{\omega}$ such that $\operatorname{supp}(F) \subseteq(-\infty, 0]$, and either $\operatorname{supp}(F)$ is infinite and $f \sim c \log x+F$, or $\operatorname{supp}(F)$ is finite and ultimately $f=c \log x+F$.

(b) $(\overline{\mathbb{R}}, \sin f)$ defines $\left(e^{\pi / c}\right)^{\mathbb{Z}}$. 
(c) If every subset of $\mathbb{R}$ definable in $(\overline{\mathbb{R}}, \sin f)$ either has interior or is nowhere dense, then $(\overline{\mathbb{R}}, \sin f)$ has field of exponents $\mathbb{Q}$ and $F \in \mathbb{R}\left(\left(x^{\mathbb{Q}}\right)\right)_{\omega}$.

Proof. Observe that $\sin f(t)=0$ iff $\frac{1}{\pi} f(t) \in \mathbb{Z}$; then apply the Monotonicity Theorem, 2.1 and Proposition 1.11 .

Since Theorem A is not vacuous (1.10), neither is Theorem 2.2 The same difficulties noted in 1.13 arise in trying to formulate appropriate sufficiency conditions.

There is an equivalent formulation of Theorem 2.2 that is perhaps more geometrically appealing. Let $f$ be as assumed in Theorem 2.2 . For $R>0$, put $\Gamma_{R}=\left\{\left(\frac{1}{x} \cos f(x), \frac{1}{x} \sin f(x)\right): x>R\right\}$. Note that $\Gamma_{R}$ spirals infinitely around and into the origin as $x \rightarrow+\infty$. Suppose there exists $R>0$ such that $\left(\overline{\mathbb{R}}, \Gamma_{R}\right)$ does not define $\mathbb{N}$. After increasing $R$, we may take $f$ to be injective, so for each $x>R,\left(\frac{1}{x} \cos f(x), \frac{1}{x} \sin f(x)\right)$ is the unique point in $\Gamma_{R}$ having Euclidean norm $1 / x$. Hence, $\sin f\left\lceil(R, \infty)\right.$ is definable in $\left(\overline{\mathbb{R}}, \Gamma_{R}\right)$, so $f$ is as in the conclusion of Theorem 2.2,

\section{EXPANDING BY NON-OSCILLATORY SEQUENCES}

In this section, we establish a stronger version of Theorem B. In brief, we first weaken the assumption that $(\overline{\mathbb{R}}, f)$ be o-minimal, and then derive a more detailed statement.

A Hardy field is a differential ordered field of germs at $+\infty$ of ultimately differentiable real functions, with the derivative of a germ being the germ of the derivative, and $f<g$ iff ultimately $f(t)<g(t)$. See e.g. [16, 17] for basic facts. We denote the germ of a function $f$ by just $f$. For $0<f \in \mathcal{H}$, we write $\Delta \log f$ instead of $\Delta(\log f)$ if no ambiguity arises.

I say that a Hardy field $\mathcal{H}$ is $\Delta$-closed if $\Delta f \in \mathcal{H}$ for every $f \in \mathcal{H}$, equivalently, if $\mathcal{H}$ is closed under $f \mapsto f(x+1)$.

Throughout this section, $\mathcal{H}$ denotes a $\Delta$-closed Hardy field containing $\mathbb{R}(x)$.

By the Monotonicity Theorem, the set of germs at $+\infty$ of the unary functions definable in a fixed o-minimal expansion of $\overline{\mathbb{R}}$ is a $\Delta$-closed Hardy field extending $\mathbb{R}(x)$. Hence, Theorem $\mathrm{B}$ is an immediate consequence of

3.1. If $f \in \mathcal{H}$ is infinitely increasing, bounded above by some $e^{x^{N}}$ and $(\overline{\mathbb{R}},\langle f\rangle) \neq \mathrm{PH}$, then there exist $c, r>0$ and $P \in \mathbb{R}[x]$ such that $\left|f e^{-P}-1\right| \leq c e^{-r x}$.

The proof is deferred to the end of the section.

As already noted, Theorem B is not vacuous, so neither is the above. On the other hand, the converse fails, e.g., $(\overline{\mathbb{R}},\langle f\rangle)=\mathrm{PH}$ if $f=e^{x^{2}}\left(1+e^{-x^{3 / 2}}\right)$; this is implicit in the proof of 3.1, so I omit details. Indeed, any attempt at a converse would have to include much better control of the asymptotics of the error term $f e^{-P}-1$, but is not clear how to make this precise. Nevertheless, in trying to do so, we are lead to a more elaborate result.

For $f \in \mathcal{H} \backslash \mathbb{R}$, put

$$
\|f\|= \begin{cases}1 /\left|f-\lim _{t \rightarrow+\infty} f(t)\right|, & \text { if } \lim _{t \rightarrow+\infty} f(t) \in \mathbb{R}, \\ |f|, & \text { otherwise. }\end{cases}
$$

Note that $\|f\| \in \mathcal{H}$ and is infinitely increasing. (For every $f \in \mathcal{H}, \lim _{t \rightarrow+\infty} f(t)$ exists in $\mathbb{R} \cup\{ \pm \infty\}$.) 
3.2. Theorem. Let $f \in \mathcal{H}$ be such that $(\overline{\mathbb{R}},\langle f\rangle) \neq \mathrm{PH}$. Then there exists $F \in$ $\mathbb{R}\left(\left(x^{\mathbb{R}}\right)\right)_{\text {w }}$ such that either $\operatorname{supp}(F)$ is infinite and $f \sim F\left(e^{x}\right)$, or $\operatorname{supp}(F)$ is finite and exactly one of the following holds:

(1) $f=F\left(e^{x}\right)$;

(2) $f-F\left(e^{x}\right) \notin \mathbb{R}$ and $\left\|f-F\left(e^{x}\right)\right\|>e^{x^{n}}$ for every $n \in \mathbb{N}$;

(3) $f-F\left(e^{x}\right) \notin \mathbb{R}$ and $\left|\left\|f-F\left(e^{x}\right)\right\| e^{-P}-1\right| \leq c e^{-r x}$ for some $c, r>0$ and $P \in \mathbb{R}[x]$ of degree at least 2 .

Proof of Theorem 3.2 from 3.1. Let $f \in \mathcal{H}$ and $d$ be the supremum of all $n$ such that $f \sim F\left(e^{x}\right)$ for some $F \in \mathbb{R}\left(\left(x^{\mathbb{R}}\right)\right)_{\omega}$ with $\operatorname{card}(\operatorname{supp}(F))=n$. We are done if $d=\infty$, so suppose that $d \in \mathbb{N}$ and $f \sim F\left(e^{x}\right)$ for some $F \in \mathbb{R}\left(\left(x^{\mathbb{R}}\right)\right)_{\omega}$ with $\operatorname{card}(\operatorname{supp}(F))=d$. If $f=F\left(e^{x}\right)$, then we are done, so suppose that $f \neq F\left(e^{x}\right)$. By 1.8, we may replace $f$ by $f-F\left(e^{x}\right)$, that is, we may assume that $d=0$ and $f \neq 0$; then $f \notin \mathbb{R}$. By replacing $f$ with $\|f\|$, we may assume that $f$ is infinitely increasing. If $f>e^{x^{n}}$ for all $n \in \mathbb{N}$, then we are done, so suppose otherwise. By 3.1. there exist $c, r>0$ and $P \in \mathbb{R}[x]$ such that $\left|f e^{-P}-1\right| \leq c e^{-r x}$. Since $d=0$, we have $\operatorname{deg} P \geq 2$.

No part of Theorem 3.2 is vacuous: Examples of each of the asymptotic behaviors listed in the conclusion of Theorem 3.2 are easily obtained from 1.9 . As for formulating sufficiency conditions, all difficulties noted earlier in this paper are still present. Moreover, the naive converse to (2) fails: Put $f=(1+1 / x) 2^{2^{x}}$ and observe that $(\overline{\mathbb{R}},\langle f\rangle)$ defines $\langle\sqrt{f(x+1)} / f\rangle$, so $(\overline{\mathbb{R}},\langle f\rangle)=\mathrm{PH}(c f$. 1.9(b)). Now, 1.9(c) does yield a partial converse to (2): If $\phi$ is any function such that

$$
\lim _{k \rightarrow+\infty}[\log \phi(k+1) / \log \phi(k)]=+\infty
$$

then $(\overline{\mathbb{R}},\langle\phi\rangle)$ is d-minimal, but this has nothing at all to do with Hardy fields. The case that $x^{n}<\log f<e^{x / n}$ for all $n \geq 1$ is the least understood at present; indeed, I have no examples where much can be said beyond that $(\overline{\mathbb{R}},\langle f\rangle)$ defines infinite closed discrete sets.

Next, we collect some consequences of Theorem 3.2

3.3. Corollary. If $f \in \mathcal{H} \backslash \mathbb{R}$ and $(\overline{\mathbb{R}},\langle f\rangle)$ defines no nontrivial cyclic subgroups of $\left(\mathbb{R}^{>0}, \cdot\right)$, then $\|f\|>e^{x^{n}}$ for each $n$.

Proof. Every countable subset of $\mathbb{R}$ is definable in PH. Apply 1.4, 1.8 and Theorem 3.2

3.4. Corollary. If $f \in \mathcal{H}$ and every subset of $\mathbb{R}$ definable in $(\overline{\mathbb{R}},\langle f\rangle)$ either has interior or is nowhere dense, then there exists $\alpha>1$ such that the conclusion of Theorem 3.2 holds with $F \in \mathbb{R}\left(\left(x^{\mathbb{Q}}\right)\right)_{\omega}$ and $e^{x}$ replaced uniformly by $\alpha^{x}$.

Proof. By Corollary 3.3 , there is nothing to do if $(\overline{\mathbb{R}},\langle f\rangle)$ defines no non-trivial cyclic subgroups of $\left(\mathbb{R}^{>0}, \cdot\right)$, so suppose that $(\overline{\mathbb{R}},\langle f\rangle)$ defines some $\alpha^{\mathbb{Z}}$ with $\alpha \neq 1$. Replace $F$ as in Theorem 3.2 with $F\left(x^{1 / \log \alpha}\right)$, which is then in $\mathbb{R}\left(\left(x^{\mathbb{Q}}\right)\right)_{\omega}$ by 1.5 and 1.8 .

3.5. Corollary. If $f \in \mathcal{H} \backslash \mathbb{R}$ and $\left(\overline{\mathbb{R}}, e^{x},\langle f\rangle\right) \neq \mathrm{PH}$, then $\|f\|$ is greater than every compositional iterate of $e^{x}$.

Proof. If not, there is a compositional iterate $L$ of $\log x \operatorname{such}$ that $L \circ\|f\| \leq \log x$. By 3.1 $(\overline{\mathbb{R}},\langle L \circ\|f\|\rangle)=$ PH. Note that $\left(\overline{\mathbb{R}}, e^{x},\langle f\rangle\right)$ defines $\langle L \circ\|f\|\rangle$. 
We now proceed toward the proof of 3.1 .

By [16, Cor. 1.1], there is a Hardy field extension of $\mathcal{H}$ that is closed under exponentiation and taking logarithms of positive elements; since

$$
\Delta \log x=\log (1+1 / x) \text { and } \Delta e^{x}=(e-1) e^{x},
$$

any minimal such extension is $\Delta$-closed, so we may assume that $\mathcal{H}$ is closed under exponentiation and taking logarithms and powers of positive elements.

The reader is now assumed to be familiar with the basics of Rosenlicht's treatment of Hardy fields, in particular, [17, pp. 659-662] and [18, §1]. For the most part, we use the same notation and conventions. Put $\mathcal{H}^{*}=\mathcal{H} \backslash\{0\}$, and let $\nu$ denote the canonical valuation on $\mathcal{H}\left(\right.$ with $\left.\nu(0)>\nu\left(\mathcal{H}^{*}\right)\right)$.

Since $\mathcal{H}$ is $\Delta$-closed, we have a minor extension of the usual Hardy field version of

L'Hôpital's Rule. Let $g, h \in \mathcal{H}^{*}, \nu(g) \neq 0$. If $\nu(g)<\nu(h)$, then $\nu\left(g^{\prime}\right)<\nu\left(h^{\prime}\right)$ and $\nu(\Delta g)<\nu(\Delta h)$. If $\nu(h) \neq 0$, then $\nu(g) \leq \nu(h)$ iff $\nu\left(g^{\prime}\right) \leq \nu\left(h^{\prime}\right)$ iff $\nu(\Delta g) \leq \nu(\Delta h)$.

(The proof is an exercise.)

If $f \in \mathcal{H}^{*}$ and $\nu(f) \neq 0$, then $\mathrm{Cl}(f)$ denotes the comparability class of $f$, that is, the set of all $g \in \mathcal{H}^{*}$ such that $\nu(g) \neq 0$ and there exist $m, n$ such that $\|f\| \leq\|g\|^{m}$ and $\|g\| \leq\|f\|^{n}$. The collection of all comparability classes of $\mathcal{H}$ is linearly ordered by $\mathrm{Cl}(f)<\mathrm{Cl}(g)$ iff $\|f\|^{n}<\|g\|$ for all $n$. Recall the key basic fact about comparability: If $f, g \in \mathcal{H}^{*}$ and $\nu(f), \nu(g) \neq 0$, then $\mathrm{Cl}(f) \leq \mathrm{Cl}(g)$ iff $\nu(\log |f|) \geq \nu(\log |g|)$ iff $\nu\left(f^{\prime} / f\right) \geq \nu\left(g^{\prime} / g\right)$.

3.6. Let $f \in \mathcal{H}^{*}, \nu(f) \neq 0$ and $\operatorname{Cl}(f)<\operatorname{Cl}\left(e^{x}\right)$. Then $\left(\overline{\mathbb{R}},\langle\Delta f / f\rangle,\left\langle\Delta^{2} f / \Delta f\right\rangle\right)=$ $\mathrm{PH}$, in particular, $(\overline{\mathbb{R}},\langle f\rangle)=\mathrm{PH}$.

Proof. We may assume that $f>0$. Note that $f^{\prime}, \Delta f \neq 0$. The result is obvious if $f \in \mathbb{R} . x+\mathbb{R}$, so we may assume also that $f^{\prime \prime}, \Delta^{2} f \neq 0$.

First, we show that $\nu\left(f^{\prime}\right)=\nu(\Delta f)$. We have: $\mathrm{Cl}(f)<\mathrm{Cl}\left(e^{x}\right)$ iff $\nu(\log f)>\nu(x)$ iff $\nu(\Delta \log f)>0$ iff $e^{\Delta \log f} \sim 1$ iff $f(x+1) \sim f$ iff $f^{\prime}(x+1) \sim f^{\prime}$ (by L'Hôpital). By the Mean Value Theorem, $1 \leq \Delta f / f^{\prime} \leq f^{\prime}(x+1) / f^{\prime}$. Hence, $f^{\prime} \sim \Delta f$, so $\nu\left(f^{\prime}\right)=\nu(\Delta f)$.

Now there are three cases to consider, based on the relation of $\mathrm{Cl}(f)$ to $\mathrm{Cl}(x)$.

Suppose that $\mathrm{Cl}(f)=\mathrm{Cl}(x)$, that is, $\nu(1 / x)=\nu\left(f^{\prime} / f\right)=\nu(\Delta f / f)$. Then $\nu(f / \Delta f)=\nu(x)$. By L'Hôpital, $\nu(\Delta(f / \Delta f))=0$. By AEG, $(\overline{\mathbb{R}},\langle\Delta f / f\rangle)=$ PH.

Suppose that $\mathrm{Cl}(f)<\mathrm{Cl}(x)$; then all of $\nu\left(f^{\prime \prime}\right), \nu\left((\Delta f)^{\prime}\right), \nu\left(\Delta^{2} f\right)$ are non-zero. Since $\nu\left(f^{\prime}\right)=\nu(\Delta f)$, we have by L'Hôpital both $\nu\left(f^{\prime \prime}\right)=\nu\left((\Delta f)^{\prime}\right)$ and $\nu\left(\Delta\left(f^{\prime}\right)\right)=$ $\nu\left(\Delta^{2} f\right)$. Clearly, $(\Delta f)^{\prime}=\Delta f^{\prime}$, so $\nu\left(f^{\prime \prime}\right)=\nu\left(\Delta^{2} f\right)$. By [18, Prop. 2(3)], $\nu\left(f^{\prime \prime} / f^{\prime}\right)=$ $\nu(1 / x)$, so $\nu\left(\Delta f / \Delta^{2} f\right)=\nu(x)$ and $\nu\left(\Delta\left(\Delta f / \Delta^{2} f\right)\right)=0$. By AEG, $\left(\overline{\mathbb{R}},\left\langle\Delta^{2} f / \Delta f\right\rangle\right)=$ $\mathrm{PH}$.

Finally, suppose that $\mathrm{Cl}(f)>\mathrm{Cl}(x)$. Since $\mathrm{Cl}\left(e^{x}\right)>\mathrm{Cl}(f)$ and $\nu\left(f^{\prime}\right)=\nu(\Delta f)$, we have $\nu(x)<\nu(f / \Delta f)<0$. Hence, $f / \Delta f$ is infinitely increasing and bounded above by $x$, so $\mathrm{Cl}(\Delta f / f) \leq \mathrm{Cl}(x)$. Replace $f$ by $\Delta f / f$ and argue as in the previous two cases.

We are now ready for the

Proof of 3.1. Let $f \in \mathcal{H}$ be infinitely increasing such that $(\overline{\mathbb{R}},\langle f\rangle) \neq \mathrm{PH}$ and $\nu(\log f) \geq \nu\left(x^{n}\right)$ for some $n$. We must find $r>0$ and $P \in \mathbb{R}[x]$ such that 
$\nu\left(f e^{-P}-1\right) \geq \nu\left(e^{-r x}\right)$. By 3.6, there exists $n \geq 1$ such that $\nu\left(x^{n+1}\right)<\nu(\log f) \leq$ $\nu\left(x^{n}\right)$. We proceed by induction on $n$.

Suppose that $\nu\left(x^{2}\right)<\nu(\log f) \leq \nu(x)$. Then $\nu(\log f)=\nu(x)$, for if not, then $\nu(x)<\nu(\Delta \log f)<0$ by L'Hôpital, so $\mathrm{Cl}\left(e^{\Delta \log f}\right)<\mathrm{Cl}(x)$, contradicting 3.6. Hence, there exists $b>0$ such that $\log f \sim b x$. Put $g=f e^{-b x}$; then $g \in \mathcal{H}^{*}$, and either $\nu(g)=0$ or $\mathrm{Cl}(g)<\mathrm{Cl}\left(e^{x}\right)$. Routine computation shows that both $\langle\Delta g / g\rangle$ and $\left\langle\Delta^{2} g / \Delta g\right\rangle$ are definable in $(\overline{\mathbb{R}},\langle f\rangle)$, so by 3.6 applied to $g$, we must have $\nu(g)=0$, that is, there exists $a>0$ such that $f \sim a e^{b x}$. Put $P=b x+\log a$. If $f=e^{P}$, then we are done, so suppose not. Put $h=1 /\left|f e^{-P}-1\right|$; then $h \in \mathcal{H}$ and is infinitely increasing. By 1.3 , $(\overline{\mathbb{R}},\langle f\rangle)$ defines $\langle h\rangle$ (since $\operatorname{deg} P=1)$. By 3.6. there exists $r>0$ such that $h \geq e^{r x}$, that is, $\left|f e^{-P}-1\right| \leq e^{-r x}$.

Assume the result for a certain $n \geq 1$; we show it for $n+1$. Let $\nu\left(x^{n+2}\right)<$ $\nu(\log f) \leq \nu\left(x^{n+1}\right)$. By L'Hôpital, $\nu\left(x^{n+1}\right)<\nu(\Delta \log f) \leq \nu\left(x^{n}\right)$. Since $n \geq 1$, $e^{\Delta \log f}$ is infinitely increasing. Inductively, there exist $r>0$ and $Q \in \mathbb{R}[x]$ such that

$$
\nu\left(e^{-r x}\right) \leq \nu\left(e^{\Delta \log f} e^{-Q}-1\right)=\nu(\exp (\Delta \log f-Q)-1) .
$$

Since $\nu(\Delta \log f-Q)>0$, we have $\exp (\Delta \log f-Q)-1 \sim \Delta \log f-Q$. Therefore, $\nu(\Delta \log f-Q) \geq \nu\left(e^{-r x}\right)$. Choose (by basic algebra) $P \in \mathbb{R}[x]$ such that $\Delta P=Q$ and $\nu(\log f-P)>0$ (we are free in the choice of $P(0)$ ). Then

$$
\nu(\Delta(\log f-P))=\nu(\Delta \log f-Q) \geq \nu\left(e^{-r x}\right)=\nu\left(\Delta e^{-r x}\right) .
$$

By L'Hôpital, $\nu(\log f-P) \geq \nu\left(e^{-r x}\right)$. Since $f e^{-P}-1=e^{\log f-P}-1 \sim \log f-P$, we have $\nu\left(f e^{-P}-1\right) \geq \nu\left(e^{-r x}\right)$.

We can extract a lesson from the proof. Write $f=e^{P+g}$. For $n>\operatorname{deg} P$, we have $\exp \left(\Delta^{n} \log f\right)=\exp \left(\Delta^{n} g\right)$, so $(\overline{\mathbb{R}},\langle f\rangle)$ defines $\left\langle\exp \left(\Delta^{n} g\right)\right\rangle$; any attempt at formulating a converse to 3.1 must take account of this.

Remark. This section is a compromise between proving only Theorem $\mathrm{B}$-i.e., the case that $(\overline{\mathbb{R}}, f)$ is o-minimal - and attempting to work in the greatest possible generality. Much of the above does not really require working over a $\Delta$-closed Hardy field extending $\mathbb{R}(x)$, only that all sequences involved be contained in a sufficiently well-behaved collection of sequences; see [1] for one possible approach.

\section{REFERENCES}

[1] M. Boshernitzan, Discrete "orders of infinity", Amer. J. Math. 106 (1984), no. 5, 1147-1198. MR0761583 (86f:12002b)

[2] L. van den Dries, A generalization of the Tarski-Seidenberg theorem, and some nondefinability results, Bull. Amer. Math. Soc. (N.S.) 15 (1986), no. 2, 189-193. MR0854552 (88b:03048)

[3] - Tame topology and o-minimal structures, London Mathematical Society Lecture Note Series, vol. 248, Cambridge University Press, Cambridge, 1998. MR1633348(99j:03001)

[4] - o-minimal structures and real analytic geometry, Current developments in mathematics, 1998 (Cambridge, MA), 1999, pp. 105-152. MR.1772324 (2001j:03075)

[5] L. van den Dries and C. Miller, Geometric categories and o-minimal structures, Duke Math. J. 84 (1996), no. 2, 497-540. MR1404337 (97i:32008)

[6] L. van den Dries and P. Speissegger, The real field with convergent generalized power series, Trans. Amer. Math. Soc. 350 (1998), no. 11, 4377-4421. MR.1458313 (99a:03036)

[7] - The field of reals with multisummable series and the exponential function, Proc. London Math. Soc. (3) 81 (2000), no. 3, 513-565. MR.1781147 (2002k:03057)

[8] H. Friedman and C. Miller, Expansions of o-minimal structures by fast sequences, J. Symbolic Logic 70 (2005), no. 2, 410-418. MR2140038 
[9] A. Kechris, Classical descriptive set theory, Graduate Texts in Mathematics, vol. 156, Springer-Verlag, New York, 1995. MR.1321597 (96e:03057)

[10] C. Miller, Expansions of the real field with power functions, Ann. Pure Appl. Logic 68 (1994), no. 1, 79-94. MR.1278550 (95i:03081)

[11] _ Exponentiation is hard to avoid, Proc. Amer. Math. Soc. 122 (1994), no. 1, 257-259. MR1195484 (94k:03042)

[12] , Tameness in expansions of the real field, Logic Colloquium '01 (Vienna, 2001), Lecture Notes Log., vol. 20, Assoc. Symbol. Logic, Urbana, IL, pp. 281-316. MR2143901

[13] C. Miller and P. Speissegger, Pfaffian differential equations over exponential o-minimal structures, J. Symbolic Logic 67 (2002), no. 1, 438-448. MR.1889560(2003a:03056)

[14] C. Miller and J. Tyne, Expansions of o-minimal structures by iteration sequences, Notre Dame J. Formal Logic, to appear.

[15] J.-P. Rolin, P. Speissegger, and A. Wilkie, Quasianalytic Denjoy-Carleman classes and o-minimality, J. Amer. Math. Soc. 16 (2003), no. 4, 751-777. MR1992825 (2004g:14065)

[16] M. Rosenlicht, Hardy fields, J. Math. Anal. Appl. 93 (1983), no. 2, 297-311. MR0700146 (85d:12001)

[17] , The rank of a Hardy field, Trans. Amer. Math. Soc. 280 (1983), no. 2, 659-671. MR0716843 (85d:12002)

[18] - Growth properties of functions in Hardy fields, Trans. Amer. Math. Soc. 299 (1987), no. 1, 261-272. MR:0869411 (88b:12010)

Department of Mathematics, The Ohio State University, 231 West 18th Avenue, Columbus, Оhiо 43210

E-mail address: miller@math.ohio-state.edu

URL: http://www.math.ohio-state.edu/ miller 